\title{
Luminescent silicon nanocrystals produced by near-infrared nanosecond pulsed laser ablation in water
}

\author{
L. Vaccaro, L. Sciortino, F. Messina, G. Buscarino, S. Agnello, M. Cannas* \\ Dipartimento di Fisica e Chimica, Università di Palermo, Via Archirafi 36, I-90123 Palermo, Italy
}

\section{A R T I C L E I N F O}

\section{Article history:}

Received 12 June 2013

Received in revised form

26 December 2013

Accepted 9 January 2014

Available online 21 January 2014

\section{Keywords:}

Laser ablation

Si-nanocrystal

Silica

Core-shell

Time-resolved luminescence

Atomic force microscopy

Micro-Raman

IR absorption

\begin{abstract}
A B S T R A C T
We report the investigation of luminescent nanoparticles produced by ns pulsed Nd:YAG laser ablation of silicon in water. Combined characterization by AFM and IR techniques proves that these nanoparticles have a mean size of $\sim 3 \mathrm{~nm}$ and a core-shell structure consisting of a Si-nanocrystal surrounded by an oxide layer. Time resolved luminescence spectra evidence visible and UV emissions; a band around $1.9 \mathrm{eV}$ originates from Si-nanocrystals, while two bands centered at $2.7 \mathrm{eV}$ and $4.4 \mathrm{eV}$ are associated with oxygen deficient centers in the $\mathrm{SiO}_{2}$ shell.
\end{abstract}

(c) 2014 Elsevier B.V. All rights reserved.

\section{Introduction}

The observation of photoluminescence $(\mathrm{PL})$ in the visible is one of the most relevant effects induced by the reduction of Si down to nanoscale [1]. This feature, surprising for a not highly emissive bulk material, has attracted a wide interest motivated by the use in several application fields (optoelectronics, photovoltaics, bioimaging) [2-5], and has led the research towards the development of production methods able to finely control the physical and chemical properties of the nanostructured Si. To this purpose, laser ablation (LA) in liquids is particularly promising since it provides effective control parameters (laser photon energy, fluence, pulse duration, liquid reactivity) for the morphology and the structure of related products and, therefore, it has attracted much attention [6-10]. Previous works have demonstrated the viability of a green method to produce Si-nanocrystals (Si-NCs) by LA in water, and have addressed their luminescence properties [11-14]. Recently, it has also been achieved the bio-functionalization of LA induced Si-NCs thus opening the way to useful applications such as fluorescence imaging or drug delivery $[15,16]$. These studies evidence a rich phenomenology of emission features, dependent both on

\footnotetext{
* Corresponding author. Tel.: +39 091 23891708; fax: +39 0916162461.

E-mail address: marco.cannas@unipa.it (M. Cannas).
}

the laser parameters and on the sample history (aging, oxidation effects, filtering treatments); however, the microscopic origin of the observed PL bands has not been clarified yet. One of the most debated questions concerns with the interplay between the intrinsic PL related to Si-NC and extrinsic PL associated with defects localized at the interface between Si and its environment [17]. To this aim, the combined use of a tunable excitation laser source and time resolved spectroscopy is useful to get a thorough understanding of the excitation/emission pathway: (i) to distinguish between electron hole radiative recombination processes and transitions localized at defect levels); (ii) to characterize the decay kinetics from the excited states. In the present work we investigate the PL of nanoparticles produced by LA of $\mathrm{Si}$ wafer in water using the first harmonic of a ns pulsed Nd:YAG laser. Our experimental approach is based on the use of time-resolved luminescence and complementary techniques to probe the morphological and structural properties, thus providing a solid support to discuss the origin of the observed PL bands.

\section{Experimental methods}

A n-type Si (100) wafer, with thickness of $0.5 \mathrm{~mm}$, was used as target. It was ultrasonically rinsed in ethanol for $30 \mathrm{~min}$ and then immersed in $8 \mathrm{~mL}$ of de-ionized water. The first harmonic of a pulsed Nd:YAG laser (Quanta System SYL 201, $\lambda=1064 \mathrm{~nm}$, 
repetition rate $10 \mathrm{~Hz}$, pulse width $5 \mathrm{~ns}$ ) was employed to irradiate the target at room temperature for $30 \mathrm{~min}$. After focusing by a lens (150 $\mathrm{mm}$ focal length), the laser spot diameter on the target was $\sim 1 \mathrm{~mm}$, and its energy density was $\sim 0.6 \mathrm{~J} / \mathrm{cm}^{2}$ per pulse. During the laser irradiation a rotator stage moved the Si target thus avoiding the crater formation on its surface.

The as-prepared solution was placed in a quartz cuvette for the optical absorption (OA) and PL measurements, while a droplet $(\sim 50 \mu \mathrm{L})$ was used for morphology and structure analysis. All the experiments to characterize the obtained solution were performed at room temperature within a day after ablation. OA measurements were carried out by a double beam spectrometer (JASCO V-560) with bandwidth of $5 \mathrm{~nm}$; the spectra were corrected by subtracting the contribution of the cuvette and water. Time resolved PL spectra were carried out under a tunable laser excitation, provided by an optical parametric oscillator pumped by the III harmonic $(3.49 \mathrm{eV})$ of a Nd:YAG laser (pulse width $\sim 5 \mathrm{~ns}$, repetition rate $10 \mathrm{~Hz}$ ). The emitted light was spectrally resolved by a grating ( 150 grooves $\mathrm{mm}^{-1}$ and $300 \mathrm{~nm}$ blaze), and then acquired by an intensified CCD camera driven by a delay generator (PI MAX Princeton Instruments) setting the acquisition time window, $W_{T}$, and the delay, $T_{D}$, with respect to the arrival of laser pulses. All the emission spectra were detected with a bandwidth of $10 \mathrm{~nm}$ and corrected for the monochromator dispersion. The photoluminescence excitation (PLE) spectra were measured manually point-by-point by tuning the laser and recording the PL intensity. Each point is normalized to the laser-pulse energy; its value is affected by an uncertainty of $\sim 10 \%$ mainly due to the power laser fluctuations. Atomic force microscopy (AFM) measurements were performed in the tapping mode by a Multimode V (Veeco Metrology) scanning probe microscope. PointProbe Plus Silicon probes were used with Al backside reflex coating, resonance frequency of $300 \mathrm{kHz}$ and a tip radius $<10 \mathrm{~nm}$. All the images were acquired on the dried droplet deposited onto a mica substrate by a scan window of $2 \mu \mathrm{m} \times 2 \mu \mathrm{m}$. Since the de-ionized water contains a limited number of particles of unknown origin with diameter of $\sim 1 \mathrm{~nm}$, in the study of the size distribution of the nanoparticles produced by LA, all the objects with diameters lower than $\sim 2 \mathrm{~nm}$ were systematically rejected. Confocal microscopy-Raman spectra were performed by a (Bruker SENTERRA) spectrometer $(\lambda=532 \mathrm{~nm}$, power $\sim 20 \mathrm{~mW})$, supplied with a CCD camera and a microtranslation stage, the spectral resolution being $3 \mathrm{~cm}^{-1}$. Infrared (IR) absorption was measured by a Fourier-transform spectrometer (Bruker Vertex 70), with spectral resolution of $0.5 \mathrm{~cm}^{-1}$. The spectra were obtained as difference between the absorption detected in a pellet (thickness of $1 \mathrm{~mm}$ ) of
LA induced nanoparticles dispersed in $\mathrm{KBr}$ powder and a reference of $\mathrm{KBr}$.

\section{Results and discussion}

Morphologic properties of LA induced nanoparticles from a $\mathrm{Si}$ target in water are displayed by the AFM image of Fig. 1. Si-NCs are dispersed, their size distribution, obtained by counting $\sim 250$ particles, is reported in the histogram: it is peaked around $\sim 3 \mathrm{~nm}$, the half width at half maximum (HWHM) is $\sim 1 \mathrm{~nm}$. This finding is in good agreement with the sizes of single Si-NCs measured by high resolution transmission electron microscope (TEM) after LA performed at $\lambda=1064 \mathrm{~nm}$ with energy density of $\sim 4.7 \mathrm{~J} / \mathrm{cm}^{2}[13]$. However, we note that in those experiments the Si-NCs appear to be aggregates of 10-20 nm; the difference with our sample, consisting of non-aggregated Si-NCs, could be due to the lower energy density $\left(\sim 0.6 \mathrm{~J} / \mathrm{cm}^{2}\right)$ used in the present work.

The structural and optical properties of these nanoparticles are shown in Fig. 2. Si-NCs are identified by the Raman line at $518 \mathrm{~cm}^{-1}$, Fig. 2(a); its redshift $\left(\Delta v=-2.2 \pm 0.2 \mathrm{~cm}^{-1}\right)$ and broadening respect to the lineshape related to a bulk $\mathrm{Si}$ crystal agree with the phonon confinement effect [18]. The dependence of the peak shift on the Si-NC size ( $d$ in $\mathrm{nm}$ ) is given by the following phenomenological law [19]:

$\Delta v=-\frac{19.856}{d^{1.586}}$

So, we can indirectly measure the mean size that results to be $\sim 4.0 \mathrm{~nm}$ and agrees with the size distribution obtained by AFM analysis. The IR absorption in Fig. 2(b) evidences three bands characteristics of amorphous $\mathrm{SiO}_{2}$ (silica). They are indeed assigned to the $\mathrm{Si}-\mathrm{O}-\mathrm{Si}$ bond-rocking $\left(\sim 470 \mathrm{~cm}^{-1}\right)$, Si-O-Si bondbending $\left(\sim 800 \mathrm{~cm}^{-1}\right)$ and $\mathrm{Si}-\mathrm{O}-\mathrm{Si}$ asymmetric bond-stretching $\left(\sim 1100 \mathrm{~cm}^{-1}\right)$ [20]. These findings agree with the oxidation of SiNC surface and the formation of a $\mathrm{Si} / \mathrm{SiO}_{2}$ core-shell structure [5]. The absorption spectrum in the visible and UV range is shown in Fig. 2(c): it increases monotonically with photon energy and evidences a shoulder below $3.0 \mathrm{eV}$. In agreement with previous studies [5], these features provide further support to the fabrication of Si-NCs.

The PL spectrum of Fig. 3(a) shows a band peaked at $1.95 \pm 0.04 \mathrm{eV}$ with a FWHM of $0.57 \pm 0.05 \mathrm{eV}$. Its decay kinetics, monitored at $1.95 \mathrm{eV}$ and displayed in the inset of Fig. 3(a), occurs in the $\mu \mathrm{s}$ timescale. The lifetime, measured as the time necessary to reduce the PL intensity by a factor of $e$, is $\tau=10 \pm 1 \mu \mathrm{s}$. The PLE
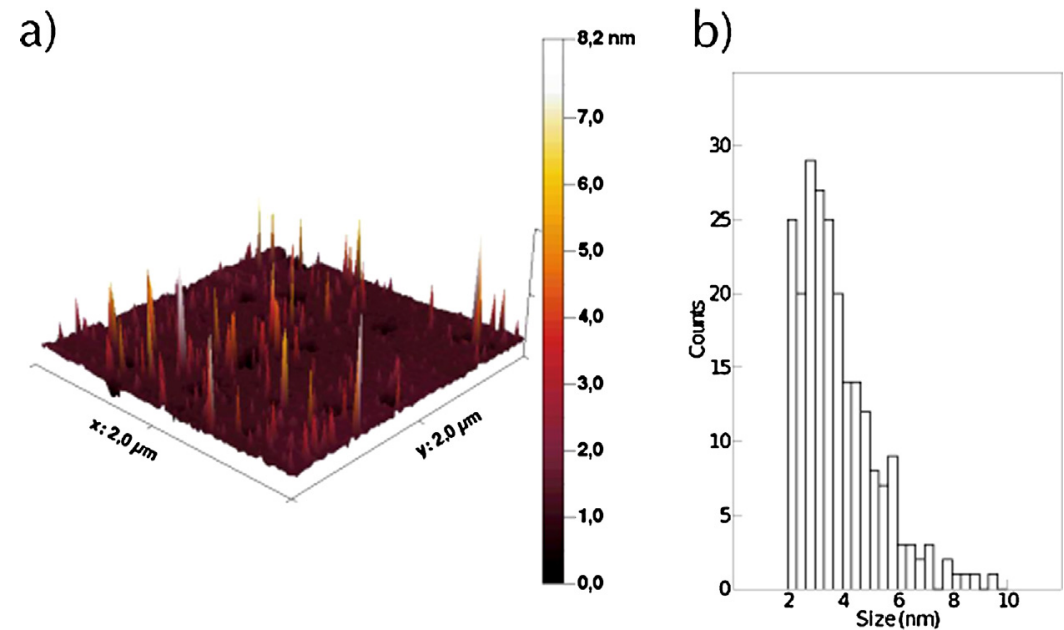

Fig. 1. AFM topographic image $(2 \mu \mathrm{m} \times 2 \mu \mathrm{m}$ scan) of LA-produced nanoparticles (a) and their size distribution (b). 

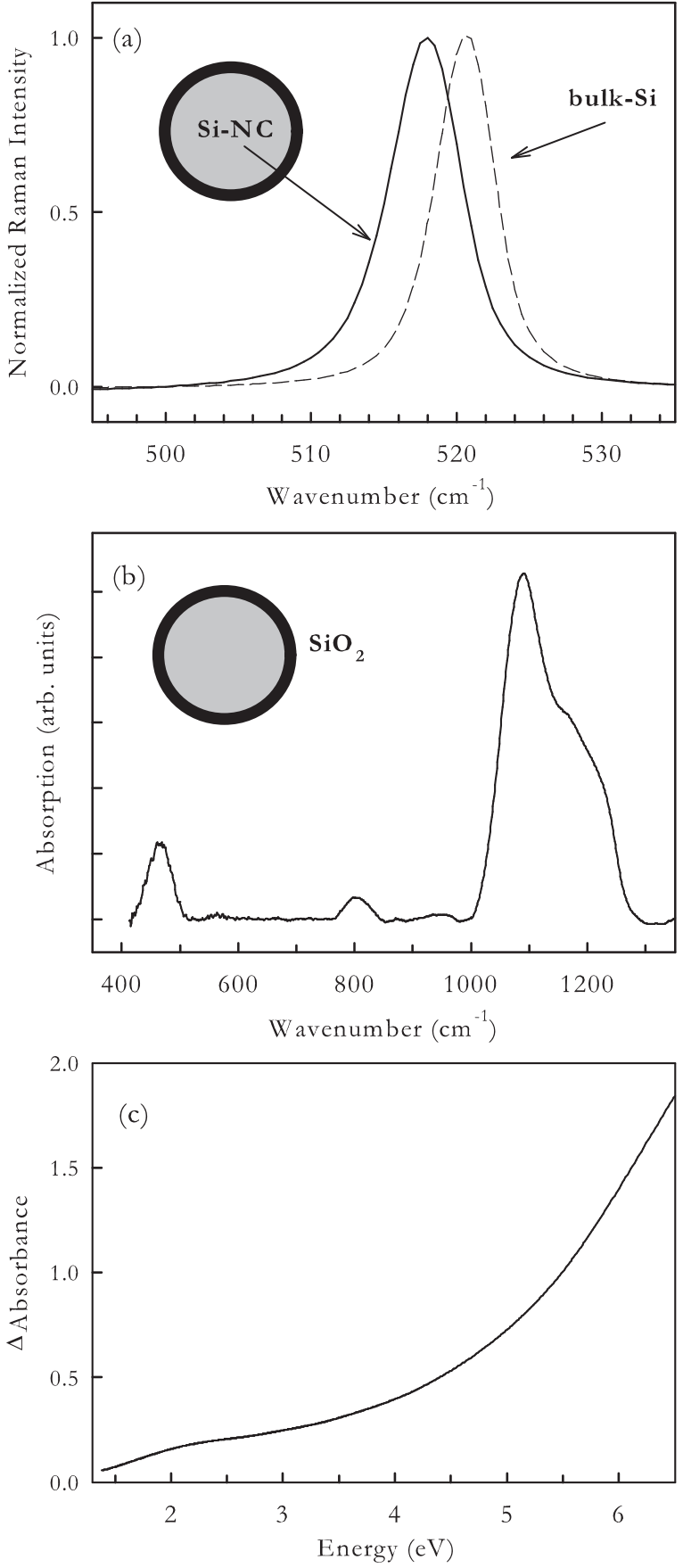

Fig. 2. (a) Raman spectra detected in the nanoparticles produced by LA (solid line) and in a bulk Si sample (dashed line). IR (b) and visible-UV (c) absorption spectrum of $\mathrm{LA}$ induced nanoparticles. The core $(\mathrm{Si}-\mathrm{NC})-\operatorname{shell}\left(\mathrm{SiO}_{2}\right)$ structure is depicted for the sake of clarity.

spectrum shown in Fig. 3(b) evidences that the emission at $1.95 \mathrm{eV}$ can be excited throughout the visible and UV range, consistently with a band-to-band transition. We observe that the origin of this PL is actually debated between two models: quantum confinement (QC) of excitons in the $\mathrm{Si}-\mathrm{NC}$ or localized states at the $\mathrm{Si}-\mathrm{SiO}_{2}$ interface [17]. In particular, it has been evidenced that in small Si-NCs ( $\operatorname{size} \sim 3 \mathrm{~nm}$ ) surface oxidation produces localized states at $\mathrm{Si}-\mathrm{O}-\mathrm{Si}$ bridge or $\mathrm{Si}=\mathrm{O}$ double bonds which favor the enhancement of the red emission $[21,22]$. Also the doping is expected to influence the PL properties; a recent work has indeed pointed out that $n-$ and p-type codoping introduces donor and acceptor states that lower the PL energy transition [23].
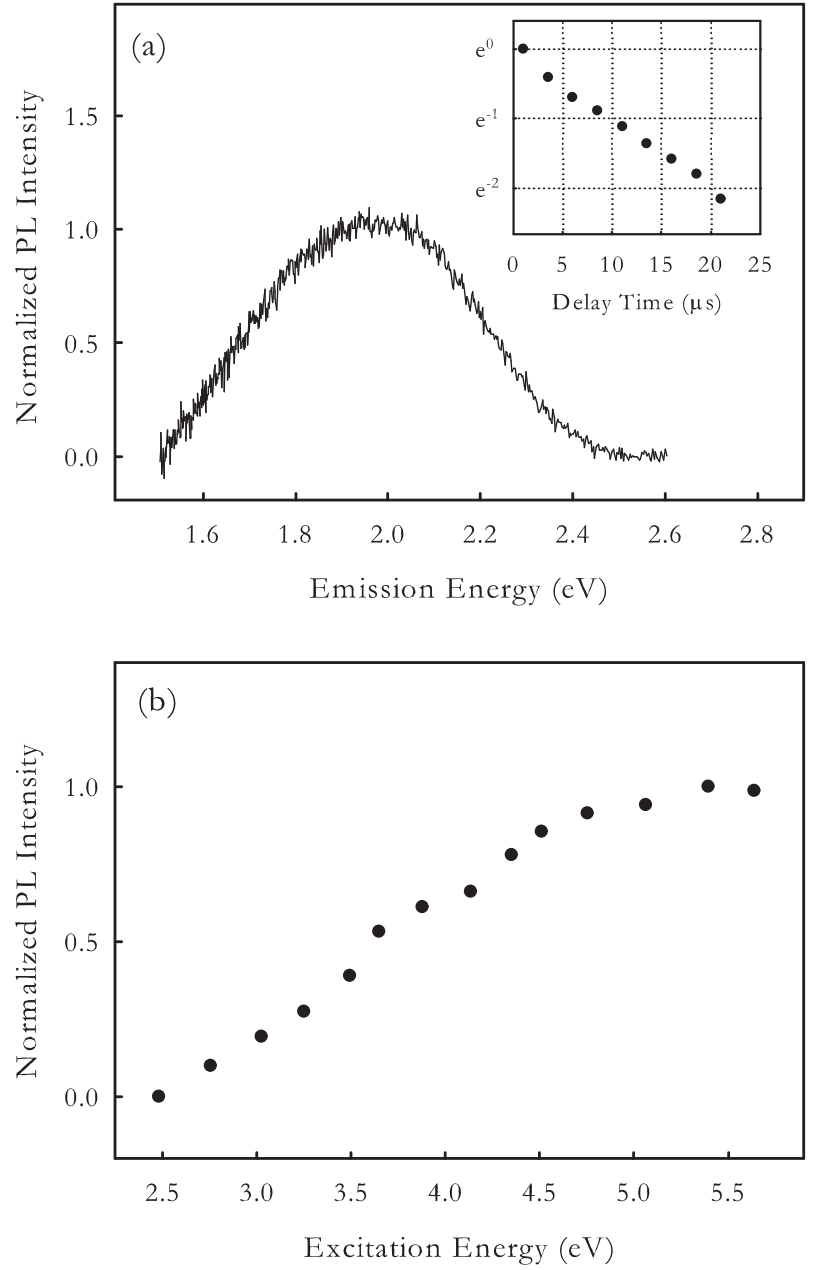

Fig. 3. (a) Time resolved PL spectrum acquired under $3.50 \mathrm{eV}$ excitation acquired with $W_{T}=100 \mu \mathrm{s}$ and $T_{D}=1 \mu \mathrm{s}$; the inset shows the decay curve monitored at $1.9 \mathrm{eV}$. (b) Excitation energy dependence of the PL amplitude measured at the peak.

Regardless its origin, we note that the peak position at $1.95 \mathrm{eV}$ agrees with the QC effect. It is well known that when the sizes of $\mathrm{Si}$-NC are smaller than $5 \mathrm{~nm}$, the band gap increases thus causing a blue shift of the PL from the IR to the visible. The PL peak position depends on the Si-NC size ( $d$ in $\mathrm{nm}$ ) by the following power law $[24,25]$ :

$E_{\mathrm{PL}}=E_{0}+\frac{3.73}{d^{1.39}}$

where $E_{0}=1.17 \mathrm{eV}$ is the band gap energy of bulk Si. Also in this case, from the measured PL peak we can indirectly derive the average of Si-NC sizes being $\sim 3.2 \mathrm{~nm}$, that is in good agreement with the size distribution measured by AFM. The FWHM of $0.57 \mathrm{eV}$, also reflects the QC effect consistently with an inhomogeneous broadening due to a size distribution. A single Si-NC, probed by confocal microscopy [26], emits, in fact, a PL with FWHM of $\sim 0.16 \mathrm{eV}$. The emission lineshape from an ensemble of Si-NCs has been also calculated in a recent work [27]; the reported results support the dependence of the emission broadening on the size distribution.

As reported in Fig. 4(a), excitation at $5.0 \mathrm{eV}$ evidences two further PL bands: the first, in the blue range, is peaked at $2.70 \pm 0.04 \mathrm{eV}$ with FWHM of $0.50 \pm 0.05 \mathrm{eV}$, its lifetime is $\tau=10 \pm 1 \mathrm{~ms}$; the second is centered at $4.42 \pm 0.04 \mathrm{eV}$ with FWHM of $0.40 \pm 0.05 \mathrm{eV}$, its lifetime is $\tau=3.5 \pm 0.5 \mathrm{~ns}$. The excitation profiles are shown in Fig. 4(b): for both emissions they consist of a UV band peaked around $5.0 \mathrm{eV}$ with FWHM of $0.35 \pm 0.05 \mathrm{eV}$. These findings 

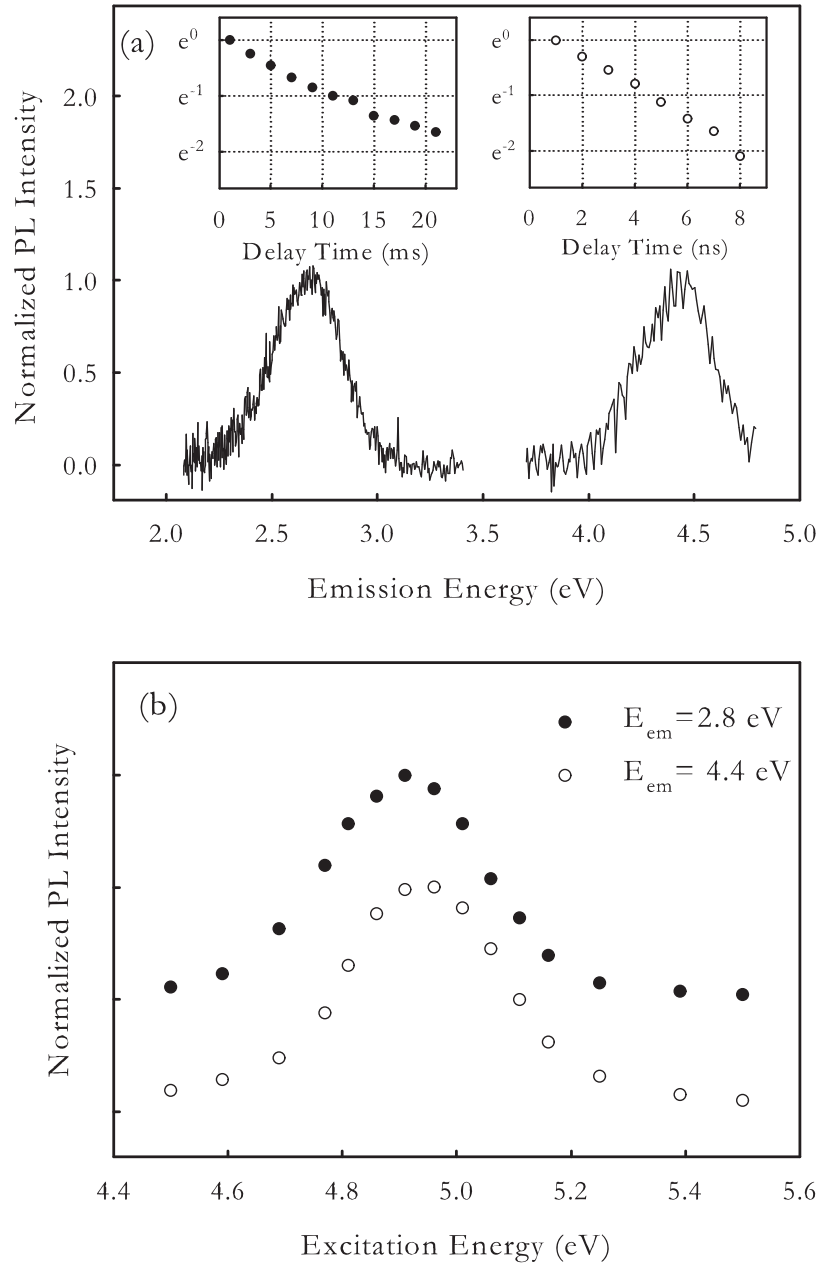

Fig. 4. (a) Time resolved PL spectra acquired under $5.00 \mathrm{eV}$ excitation, the band around $2.7 \mathrm{eV}$ is acquired with $W_{T}=10 \mathrm{~ms}$ and $T_{D}=1 \mathrm{~ms}$, the band around $4.4 \mathrm{eV}$ is acquired with $W_{T}=10 \mathrm{~ns}$ and $T_{D}=1 \mathrm{~ns}$. The decay curves of these PL emissions are shown in the insets. (b) Normalized excitation spectra monitored at $2.8 \mathrm{eV}$ and $4.4 \mathrm{eV}$; the curves are vertically shifted for the sake of clarity.

demonstrate that the excitation transition occurs between levels localized at the same defect. In particular, on the basis of the comparison with literature data, both PL bands are assigned to oxygen deficient centers in amorphous $\mathrm{SiO}_{2}$ [28]. In fact, these luminescent defects are observed in irradiated or oxygen-deficient silica, both in bulk and in porous film with high specific surface $[29,30]$. The

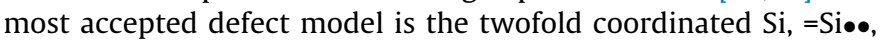
where the symbol $(\bullet \bullet)$ stands for two paired electrons [31]. In agreement with its energy level scheme, the fast $4.4 \mathrm{eV}$ PL originates from an allowed singlet-to-singlet transition, inverse of the $5.0 \mathrm{eV}$ excitation, the Stokes-shift of $0.6 \mathrm{eV}$ being due to a large electron-phonon coupling. The slow $2.7 \mathrm{eV}$ band is associated with a forbidden triplet-to-singlet transition, its excitation being due to an intersystem crossing between the excited singlet and triplet states. We acknowledge that in previous experiments on LA of Si in water, a blue emission is observed under several excitation energies in the UV range and it is ascribed to QC in Si-NC $[11,13,14]$. It is worth noting that these excitation features differ from those here evidenced (a bell-shaped band peaked at $5.0 \mathrm{eV}$ ). This comparison supports the attribution of the $2.7 \mathrm{eV}$ and $4.4 \mathrm{eV}$ PL bands to defects in the oxide layer instead of a QC effect in the Si-NCs.

\section{Conclusion}

Time-resolved PL experiments were successful to study the spectral and decay properties of the emission bands originating from a $\mathrm{Si} / \mathrm{SiO}_{2}$ core-shell nanoparticle produced by ns LA of $\mathrm{Si}$ in water. A red band $(1.9 \mathrm{eV})$ is emitted from the $\mathrm{Si}-\mathrm{NC}$ and agrees with the QC effect; a blue $(2.7 \mathrm{eV})$ and an $\mathrm{UV}(4.4 \mathrm{eV})$ band originate from a defective silica layer surrounding the $\mathrm{Si}-\mathrm{NC}$. These results are relevant in the current understanding of nontoxic luminescent Si-NCs in view of their potential applications in optics and medicine.

\section{Acknowledgements}

The work was partially supported by FAE project, PO FESR Sicilia 2007/2013 4.1.1.1. We thank the group of the Laboratory of Advanced Materials Physics (Palermo University) for their support and stimulating discussions and G. Napoli and G. Tricomi for technical assistance.

\section{References}

[1] L.T. Canham, Appl. Phys. Lett. 57 (1990) 1046.

[2] L. Pavesi, L.D. Negro, C. Mazzoleni, G. Franzò, F. Priolo, Nature 408 (2000) 440.

[3] S.K. Kim, C.H. Cho, B.H. Kim, S.J. Park, J.W. Lee, Appl. Phys. Lett. 95 (2009) 143120

[4] K.Y. Cheng, R. Anthony, U.R. Kortshagen, R.J. Holmes, Nano Lett. 11 (2011) 1952.

[5] R. Intartaglia, K. Bagga, M. Scotto, A. Diaspro, F. Brandi, Opt. Mater. Expr. 2 (2012) 510

[6] R. Intartaglia, K. Bagga, F. Brandi, G. Das, A. Genovese, E.D. Fabrizio, A. Diaspro, J. Phys. Chem. C 115 (2011) 5102.

[7] H. Zeng, X.W. Du, S.C. Singh, S.A. Kulinich, S. Yang, J. He, W. Cai, Adv. Funct Mater. 22 (2012) 1333

[8] R. Intartaglia, K. Bagga, A. Genovese, A. Athanassiou, R. Cingolani, A. Diaspro, F, Brandi, Phys. Chem. Chem. Phys. 14 (2012) 15406

[9] P. Blandin, K.A. Maximova, M.B. Gongalsky, J.F. Sanchez-Royo, V.S. Chirvony, M Sentis, V.Y. Timoshenkob, A.V. Kabashin, J. Mater. Chem. B 1 (2013) 2489.

[10] D.M. Popovic, J.S. Chai, A.A. Zekic, M. Trtica, M. Momcilovic, S. Maletic, Laser Phys. Lett. 10 (2013) 026001

[11] V. Švrček, T. Sasaki, Y. Shimizu, N. Koshizaki, Appl. Phys. Lett. 89 (2006) 213113.

[12] X.W. Du, W.J. Qin, Y.W. Lu, X. Han, Y.S. Fu, S.L. Hu, J. Appl. Phys. 102 (2007) 013518.

[13] S. Yang, W. Cai, H. Zeng, Z. Li, J. Appl. Phys. 104 (2008) 023516.

[14] S. Alkis, A.K. Okyay, B. Ortaç, J. Phys. Chem. C 116 (2012) 3432

[15] R. Intartaglia, A. Barchanski, K. Bagga, A. Genovese, G. Das, P. Wagener, E.D. Fabrizio, A. Diaspro, F. Brandi, S. Barcikowski, Nanoscale 4 (2012) 1271.

[16] K. Bagga, A. Barchanski, R. Intartaglia, S. Dante, R. Marotta, A. Diaspro, C.L. Sajti, F. Brandi, Laser Phys. Lett. 10 (2013) 065603.

[17] S. Godefroo, M. Hayne, M. Jivanescu, A. Stesmans, M. Zacharias, O.I. Lebedev G.V. Tendeloo, V.V. Moshchalkov, Nat. Nanotechnol. 3 (2008) 174

[18] P.G. Kuzmin, G.A. Shafeev, V.V. Bukin, S.G. Garnov, C. Farcau, R. Carles, B. WarotFontrose, V. Guieu, G. Viau, J. Phys. Chem. C 114 (2010) 15266

[19] J. Zi, K. Zhang, X. Xie, Phys. Rev. B 55 (1997) 023516.

[20] A. Agarwal, M. Tomozawa, J. Non-Cryst. Solids 209 (1997) 166.

[21] W.Q. Huang, Z.M. Huang, H.Q. Cheng, X.J. Miao, Q. Shu, S.R. Liu, C.J. Qin, Appl. Phys. Lett. 101 (2012) 171601.

[22] K. Dohnaloá, A.N. Poddubny, A.A. Prokofiev, W.D. de Boer, C.P. Umesh, J.M. Paulusse, H. Zuilhof, T. Gregorkiewicz, Light Sci. Appl. 2 (2013) e47.

[23] H. Sugimoto, M. Fujii, K. Imakita, S. Hayashi, K. Akamatsu, J. Phys. Chem. C 117 (2013) 11850.

[24] G. Ledoux, O. Guillois, D. Porterat, C. Reynaud, F. Huisken, B. Kohn, V. Paillard, Phys. Rev. B 62 (2000) 15942

[25] A. Gupta, H. Wiggers, Nanotechnology 22 (2011) 055707.

[26] T. Schmidt, A.I. Chizhik, A.M. Chizhik, K. Potrick, A.J. Meixner, F. Huisken, Phys Rev. B 86 (2012) 125302

[27] R. Guerra, F. Cigarini, S. Ossicini, J. Appl. Phys. 113 (2013) 143505.

[28] L. Skuja, J. Non-Cryst. Solids 239 (1998) 16.

[29] V.N. Bagratashvili, S.I. Tsypina, V.A. Radzig, A.O. Rybaltovskii, P.V. Chernov, S.S. Alimpiev, Y.O. Simanovskii, J. Non-Cryst. Solids 180 (1995) 221.

[30] R. Boscaino, M. Cannas, F.M. Gelardi, M. Leone, Phys. Rev. B 54 (1996) 6194.

[31] L. Skuja, J. Non-Cryst. Solids 149 (1992) 77. 